\title{
On fixed point theory in topological posets, extended quasi-metric spaces and an application to asymptotic complexity of algorithms
}

\author{
Naseer Shahzad ${ }^{1 *}$, Oscar Valero², Mohammed A Alghamdi and Maryam A Alghamdi
}

"Correspondence:
nshahzad@kau.edu.sa
1Operator Theory and Applications
Research Group, Department of
Mathematics, King Abdulaziz
University, P.O. Box 80203, Jeddah,
21859 , Saudi Arabia
Full list of author information is
available at the end of the article

\begin{abstract}
In this paper we present a few fixed point results in the framework of topological posets. To this end, we introduce an appropriate notion of completeness and order-continuity. Special attention is paid to the case that the topology of the topological poset is induced by an extended quasi-metric. Finally, the applicability of the exposed results is illustrated providing a methodology to determine the asymptotic upper bound of the complexity of those algorithms whose running time of computing is the solution to a special type of recurrence equation.
\end{abstract}

MSC: $06 \mathrm{A06} ; 47 \mathrm{H} 10 ; 54 \mathrm{E} 50 ; 54 \mathrm{H} 25 ; 54 \mathrm{~F} 05 ; 68 \mathrm{Q} 25$

Keywords: topological poset; extended quasi-metric; Hausdorff; monotone; fixed point; asymptotic complexity analysis

\section{Introduction}

Fixed point techniques in partially ordered sets (posets for short) have been widely used as a mathematical tool for modeling in computer science (see, for instance, [1] and [2]). In addition to the fixed point methods based on order-theoretic foundations, a few metric fixed point techniques have also been shown to be useful in a few fields of computer science (see, for example, [3]). Concretely, Banach type fixed point theorems in generalized metric spaces have been used to describe processes in logic programming $[3,4]$ and in complexity analysis ([5] and [6]). For more applications in generalized metric spaces, we refer the reader to [7-9].

Inspired by the utility of both fixed point approaches, we prove a few fixed point results in a general framework which provides a bridge between two worlds, the order-theoretic and the metric one. Concretely we give fixed point results in the general framework of topological posets (posets endowed with a topology). In order to provide the aforesaid results we introduce a new notion of completeness, which entails the order and the topology at the same time, and an appropriate notion of order-continuity. Later on, we consider the special case of those topological posets whose topology is induced by an extended quasimetric (in the sense of [6]) in such a way that several fixed point results involving Banach type contractive conditions in extended quasi-metric spaces are derived as a particular

(c) 2015 Shahzad et al. This article is distributed under the terms of the Creative Commons Attribution 4.0 International License (http://creativecommons.org/licenses/by/4.0/), which permits unrestricted use, distribution, and reproduction in any medium, provided you give appropriate credit to the original author(s) and the source, provide a link to the Creative Commons license, and indicate if changes were made. 
case. Finally, we apply, in the spirit of [6], the developed theory to complexity analysis by means of determining the asymptotic upper bound of the complexity of those algorithms whose running time of computing is the solution to a special type of recurrence equation.

In the remainder of the paper we will need a few basic notions from the theory of posets and extended quasi-metric spaces. For this reason, and for the sake of completeness, we end this section recalling them in the following.

According to [1], a partially ordered set (poset for short) is a pair $(X, \leq)$ such $X$ is a nonempty set and $\leq$ is a reflexive, antisymmetric and transitive binary relation on $X$. Moreover, if $(X, \leq)$ is a partially ordered set and $Y \subseteq X$, then a lower bound for $Y$ in $(X, \leq)$ is an element $x \in X$ such that $x \leq y$ for all $y \in Y$. The infimum for $Y$ in $(X, \leq)$, if it exists, is an element $z \in X$ which is a lower bound for $Y$ and, in addition, satisfies $x \leq z$ provided that $x \in X$ is a lower bound for $Y$. In the sequel, we will denote by $\downarrow \leq x$, with $x \in X$, the set $\{y \in X: y \leq x\}$. As usual, a sequence $\left(x_{n}\right)_{n \in \mathbb{N}}$ in $(X, \leq)$ is decreasing if $x_{n+1} \leq x_{n}$ for all $n \in \mathbb{N}$, where $\mathbb{N}$ denotes the set of positive integer numbers. As usually, a mapping $f$ from a partially ordered set $(X, \leq)$ into itself will be called monotone if $f(x) \leq f(y)$ whenever $x \leq y$.

Following [6], an extended quasi-metric $X$ on a nonempty set $X$ is a function $d: X \times X \rightarrow$ $\mathbb{R}^{+} \cup\{\infty\}$ such that for all $x, y, z \in X$ :

(i) $d(x, y)=d(y, x)=0 \Leftrightarrow x=y$.

(ii) $d(x, z) \leq d(x, y)+d(y, z)$.

Each extended quasi-metric $d$ on a set $X$ induces a $T_{0}$ topology $\tau(d)$ on $X$ which has as a base the family of open $d$-balls $\left\{B_{d}(x, r): x \in X, r>0\right\}$, where $B_{d}(x, r)=\{y \in X: d(x, y)<r\}$ for all $x \in X$ and $r>0$. Observe that the topology induced by an extended quasi-metric is only $T_{0}$ but not $T_{2}$ in general.

Of course, an extended quasi-metric space is a pair $(X, d)$ such that $X$ is a nonempty set and $d$ is an extended quasi-metric on $X$.

When an extended quasi-metric $d$ on a nonempty set $X$ holds that $d(x, y)<\infty$ for all $x, y \in X$, then it is called a quasi-metric on $X$ and, besides, the pair $(X, d)$ is called a quasimetric space (see, for instance, [10]). Obviously a metric on a set $X$ is a quasi-metric $d$ on $X$ satisfying for all $x, y \in X$ :

(iii) $d(x, y)=d(y, x)$.

\section{The mathematical results}

In this section we prove our main result which will be central in the application later on. In order to introduce the aforesaid result we need to fix a few pertinent notions.

In the following, we will say that a topological poset $(X, \tau, \leq)$ is $\leq-\tau$-complete provided that every decreasing sequence $\left(x_{n}\right)_{n \in \mathbb{N}}$ in $(X, \leq)$ has a lower bound $x$ to which it converges with respect to $\tau$.

From now on, a mapping from a topological poset $(X, \tau, \leq)$ into itself will be said to be monotone- $\leq$-continuous if, it is monotone and, in addition, given $z \in X$, then the sequence $\left(f^{n+1}(z)\right)_{n \in \mathbb{N}}$ converges to $f(x)$ with respect to $\tau$ whenever the sequence $\left(f^{n}(z)\right)_{n \in \mathbb{N}}$ is decreasing and $x$ is a lower bound of it such that $\left(f^{n}(z)\right)_{n \in \mathbb{N}}$ converges to $x$ with respect to $\tau$.

In the light of the preceding notions we are able to introduce our main result.

Theorem 1 Let $(X, \tau, \leq)$ be a $\leq-\tau$-complete topological poset and let $f$ be a mapping from $X$ into itself. Assume that the following assertions hold: 
(1) $(X, \tau)$ is Hausdorff.

(2) There exists $x_{0} \in X$ such that $f\left(x_{0}\right) \leq x_{0}$.

(3) $f$ is monotone- $\leq$-continuous.

Then $f$ has a fixed point $x^{*}$ such that $x^{*} \in \downarrow \leq x_{0}$.

Proof Let $x_{0} \in X$ such that $f\left(x_{0}\right) \leq x_{0}$. Of course we can assume without lose of generality that $x_{0} \neq f\left(x_{0}\right)$, since otherwise the proof is finished. Since $f\left(x_{0}\right) \leq x_{0}$ and $f$ is monotone we immediately see that $\left(f^{n}\left(x_{0}\right)\right)_{n \in \mathbb{N}}$ is a decreasing sequence in $(X, \leq)$. By the $\leq-\tau$ completeness of $(X, \tau, \leq)$ we deduce the existence of a lower bound $x^{*}$ of $\left(f^{n}\left(x_{0}\right)\right)_{n \in \mathbb{N}}$ in $(X, \leq)$ such that $\left(f^{n}\left(x_{0}\right)\right)_{n \in \mathbb{N}}$ converges to $x^{*}$ with respect to $\tau$. Then $x^{*} \in \downarrow_{\leq} x_{0}$. Since $f$ is monotone- $\leq$-continuous we see that $\left(f^{n}\left(x_{0}\right)\right)_{n \in \mathbb{N}}$ converges to $f\left(x^{*}\right)$ with respect to $\tau$. Thus $f\left(x^{*}\right)=x^{*}$, since $(X, \tau)$ is Hausdorff.

In the next example we show that the $\leq-\tau-$-completeness of the topological poset cannot be deleted in Theorem 1 in order to guarantee the existence of a fixed point.

Example 2 Consider the extended quasi-metric space $\left(\mathbb{R}_{0}^{+}, d_{S}\right)$, where $\mathbb{R}_{0}^{+}=\left\{x \in \mathbb{R}^{+}: x>\right.$ $0\}$ and the extended quasi-metric $d_{S}$ is defined as follows:

$$
d_{S}(x, y)= \begin{cases}y-x & \text { if } x \leq y \\ +\infty & \text { if } x>y\end{cases}
$$

where $\leq$ stands for the usual partial order on $\mathbb{R}^{+}$. Moreover, consider the partial order $\preceq$ on $\mathbb{R}_{0}^{+}$defined by $x \preceq y \Leftrightarrow y \leq x$. Of course $\left(\mathbb{R}_{0}^{+}, \tau\left(d_{S}\right), \preceq\right)$ is a topological poset such that $\left(\mathbb{R}_{0}^{+}, \tau\left(d_{S}\right)\right)$ is Hausdorff. It is clear that $\left(\mathbb{R}_{0}^{+}, \tau\left(d_{S}\right), \preceq\right)$ is not $\preceq-\tau\left(d_{S}\right)$-complete. Indeed, the sequence $\left(x_{n}\right)_{n \in \mathbb{N}}$ given by $x_{n}=1-\frac{1}{2 n}$ is decreasing in $\left(\mathbb{R}_{0}^{+}, \preceq\right)$ and has 1 a lower bound. However, $\left(x_{n}\right)_{n \in \mathbb{N}}$ does not converge to 1 with respect to $\tau\left(d_{S}\right)$. Next, define the mapping $f$ from $\mathbb{R}_{0}^{+}$into itself by $f(x)=x+1$. Clearly $f$ is monotone- $\preceq$-continuous. In addition, $f(0)=1 \preceq 0$. It is obvious that $f$ has no fixed points.

The next example shows that the Hausdorff condition of the topological poset cannot be weakened in Theorem 1 in order to guarantee the existence of a fixed point.

Example 3 Set $\mathbb{R}_{1}^{+}=\mathbb{R}^{+} \backslash\{1\}$. Define on $\mathbb{R}_{1}^{+}$the extended quasi-metric $d_{\infty}$ by

$$
d_{\infty}(x, y)= \begin{cases}0 & \text { if } x \geq y \\ +\infty & \text { if } x<y\end{cases}
$$

where $\leq$ denotes the usual partial order on $\mathbb{R}^{+}$. It is a simple matter to see that $\left(\mathbb{R}_{1}^{+}, \tau\left(d_{\infty}\right), \leq\right)$ is $\leq-\tau\left(d_{\infty}\right)$-complete. Define the mapping $f$ from $\mathbb{R}_{1}^{+}$into itself by $f(x)=\frac{x+1}{2}$ for all $x \in \mathbb{R}_{1}^{+}$. Clearly $f$ is monotone and $f(x) \leq x$ for all $x \in \mathbb{R}_{1}^{+}$with $x>1$. It is not hard to see $f$ is monotone- $\leq$-continuous. Of course, $\left(\mathbb{R}_{1}^{+}, \tau\left(d_{\infty}\right)\right)$ is not Hausdorff and $f$ has no fixed points.

The example below shows that the monotone- $\leq$-continuity of the mapping cannot be omitted in the statement of Theorem 1 in order to provide the existence of a fixed point. 
Example 4 Consider the extended quasi-metric space $\left(X, d_{S}\right)$, where $X=\{0,1\}$ and $d_{S}$ is the extended quasi-metric introduced in Example 2. Consider the partial order $\preceq$ defined on $X$ by $0 \preceq 1$. It is immediate to see that $\left(X, \tau\left(d_{S}\right), \preceq\right)$ is $\preceq-\tau\left(d_{S}\right)$-complete topological poset such that $\left(X, \tau\left(d_{S}\right)\right)$ is Hausdorff. Define the mapping $f$ from $X$ into itself by $f(0)=1$ and $f(1)=0$. Then $f(1)=0 \preceq 1$. However, $f$ is not monotone- $\preceq$-continuous because $f$ is not monotone. In fact, $f(0)$ and $f(1)$ is not $\preceq$-related although $0 \preceq 1$. Moreover, $f$ has no fixed points.

Next we show that the existence of an element $x_{0}$ such that $f\left(x_{0}\right) \leq x_{0}$ cannot be omitted in the statement of Theorem 1 to ensure the existence of a fixed point.

Example 5 Consider the extended quasi-metric space $\left(\mathbb{R}^{+}, d_{2}\right)$ defined as follows:

$$
d_{2}(x, y)= \begin{cases}y-x & \text { if } x \leq y \\ 2(x-y) & \text { if } x>y\end{cases}
$$

where again $\leq$ denotes the usual order on $\mathbb{R}^{+}$. It is obvious that $\left(\mathbb{R}^{+}, \tau\left(d_{2}\right)\right)$ is Hausdorff. It is easy to check that $\left(\mathbb{R}^{+}, \tau\left(d_{2}\right), \leq\right)$ is $\leq-\tau\left(d_{2}\right)$-complete. Define the mapping $f$ from $\mathbb{R}^{+}$into itself by $f(x)=x+1$ for all $x \in \mathbb{R}^{+}$. Clearly $f$ is monotone- $\leq$-continuous. Besides $x \leq f(x)$ for any $x \in \mathbb{R}^{+}$. Of course $f$ has no fixed points.

In the following example we show that Theorem 1 does not provide the uniqueness of the fixed point in general.

Example 6 Consider the extended quasi-metric space $\left(X, d_{S}\right)$ introduced in Example 4 . Define the partial order $\preceq$ on $X$ by $x \preceq y \Leftrightarrow x=y$. Then $\left(X, \tau\left(d_{S}\right), \preceq\right)$ is a topological poset such that $\left(X, \tau\left(d_{S}\right)\right)$ is Hausdorff. Moreover, a simple verification shows that $\left(X, \tau\left(d_{S}\right), \preceq\right)$ is $\preceq-\tau\left(d_{S}\right)$-complete. Define the mapping $f$ from $X$ into itself by $f(x)=x$ for all $x \in X$. It is obvious that $f$ is monotone- $\preceq$-continuous and that $f(x) \preceq x$ for all $x \in X$. Clearly, the set of a fixed point of $f$ matches up with $X$.

We will say that a topological poset is meet $\leq-\tau-$-complete if every decreasing sequence $\left(x_{n}\right)_{n \in \mathbb{N}}$ in $(X, \leq)$ has an infimum $x$ to which it converges with respect to $\tau$.

Corollary 7 Let $(X, \tau, \leq)$ be a meet $\leq-\tau$-complete topological poset and let $f$ be a mapping from $X$ into itself. Assume that the following assertions hold:

(1) $(X, \tau)$ is Hausdorff.

(2) There exists $x_{0} \in X$ such that $f\left(x_{0}\right) \leq x_{0}$.

(3) $f$ is monotone- $\leq$-continuous.

Then $f$ has a fixed point $x^{*}$ such that $x^{*} \in \downarrow \leq x_{0}$.

In the light of Example 6 we provide a sufficient condition, which we have called decreasing convergence property, which guarantees the uniqueness of the fixed point. Thus, from now on, we will say that a topological poset $(X, \tau, \leq)$ has the orbital decreasing convergence property with respect to a mapping $f$ from $X$ into itself provided that there exists $z \in X$ such that $\left(f^{n}(x)\right)_{n \in \mathbb{N}}$ converges to $z$ whenever $\left(f^{n}(y)\right)_{n \in \mathbb{N}}$ converges to $z$ and $x \leq y$. 
Example 8 Let $d_{S}$ be the extended quasi-metric on $\mathbb{R}^{+}$introduced in Example 2. Consider the topological poset $\left(\mathbb{R}^{+}, \tau\left(d_{S}\right), \leq\right)$, where $\leq$ stands for the usual partial order on $\mathbb{R}^{+}$. Define the mapping $f$ from $\mathbb{R}^{+}$into itself by $f(x)=\frac{x}{2}$ for all $x \in \mathbb{R}^{+}$. It is not hard to see that $\left(\mathbb{R}^{+}, \tau\left(d_{S}\right), \leq\right)$ has the orbital decreasing property with respect to $f$.

Theorem 9 Let $(X, \tau, \leq)$ be a $\leq-\tau$-complete topological poset and let $f$ be a mapping from $X$ into itself. Assume that the following assertions hold:

(1) $(X, \tau)$ is Hausdorff.

(2) There exists $x_{0} \in X$ such that $f\left(x_{0}\right) \leq x_{0}$.

(3) $f$ is monotone- $\leq$-continuous.

(4) $(X, \tau, \leq)$ has the orbital decreasing convergence property with respect to $f$.

Then $f$ has a unique fixed point in $\downarrow \leq x_{0}$.

Proof The existence of the fixed point $x^{*}$ of $f$ is guaranteed by Theorem 1. Moreover, the aforementioned result shows that $\left(f^{n}\left(x_{0}\right)\right)_{n \in \mathbb{N}}$ converges to $x^{*}$ with respect to $\tau$. Next assume that there exists $z \in X$ such that $z$ is a fixed point of $f$ and $z \in \downarrow_{\leq} x_{0}$. Then $z \leq x_{0}$. Since $\left(f^{n}\left(x_{0}\right)\right)_{n \in \mathbb{N}}$ converges to $x^{*}$ with respect to $\tau$ and $(X, \tau, \leq)$ has the orbital decreasing convergence property we deduce that $\left(f^{n}(z)\right)_{n \in \mathbb{N}}$ converges to $x^{*}$ with respect to $\tau$. Hence we deduce that $z=x^{*}$ because $f^{n}(z)=z$ for all $n \in \mathbb{N}$ and $(X, \tau)$ is Hausdorff.

The next example shows that the orbital decreasing convergence condition cannot be deleted in the statement of Theorem 9 in order to guarantee the uniqueness of the fixed point.

Example 10 Consider the topological poset $\left(X, \tau\left(d_{S}\right), \preceq\right)$ introduced in Example 4 . Then $\left(X, \tau\left(d_{S}\right), \preceq\right)$ is $\preceq-\tau\left(d_{S}\right)$-complete and, in addition, $\left(X, \tau\left(d_{S}\right)\right)$ is Hausdorff. Define the mapping $f$ from $X$ into itself by $f(x)=x$ for all $x \in X$. It is obvious that $f$ is monotone- $\preceq$ continuous and that $f(x) \preceq x$ for all $x \in X$. Nevertheless, the set of fixed points of $f$ is exactly the whole set $X$. It is clear that $\left(X, \tau\left(d_{S}\right), \preceq\right)$ does not have the orbital decreasing property with respect to $f$, since there does not exist $z \in X$ such that $\left(f^{n}(0)\right)_{n \in \mathbb{N}}$ and $\left(f^{n}(1)\right)_{n \in \mathbb{N}}$ converge to $z$ with respect to $\tau\left(d_{S}\right)$ although $0 \preceq 1$.

In the remainder of this section we introduce a few fixed point results in which the topology of the topological poset is induced by an extended quasi-metric. To this end, let us recall, according to [6], that an extended quasi-metric space $(X, d)$ is said to be balanced if, given $r, s>0$ and $x, y \in X$ we have $d(x, y) \leq r+s$ whenever there exist sequences $\left(x_{n}\right)_{n \in \mathbb{N}}$, and $\left(y_{n}\right)_{n \in \mathbb{N}}$ with $\lim _{n, m \rightarrow \infty} d\left(y_{m}, x_{n}\right)=0$ and such that $d\left(x, x_{n}\right) \leq r$ and $d\left(y_{n}, y\right) \leq s$ for all $n \in \mathbb{N}$.

Corollary 11 Let $(X, \tau, \leq)$ be a topological poset and let $d$ be an extended quasi-metric on $X$ such that $\tau=\tau(d)$ and $(X, \tau(d), \leq)$ is $\leq-\tau(d)$-complete. Assume that the following assertions hold:

(1) The extended quasi-metric space $(X, d)$ is balanced.

(2) There exists $x_{0} \in X$ such that $f\left(x_{0}\right) \leq x_{0}$.

(3) $f$ is monotone- $\leq$-continuous.

(4) $(X, \tau(d), \leq)$ has the orbital decreasing convergence property with respect to $f$.

Then $f$ has a unique fixed point in $\downarrow \leq x_{0}$. 
Proof Since $(X, d)$ is balanced we see that $(X, \tau(d))$ is Hausdorff. Thus Theorem 1 gives the existence of a fixed point $x^{*}$ such that $x^{*} \in \downarrow \leq x_{0}$. Moreover, Theorem 9 yields the uniqueness of the fixed point in $\downarrow \leq x_{0}$, since $(X, \tau(d)$, $\leq)$ has the orbital decreasing convergence property with respect to $f$.

It must be stressed that Corollary 11 is related to Proposition 2 and Theorem 1 in [11], where fixed point results for expanding mappings defined from partially ordered complete metric spaces into itself have been proved. In fact, from Corollary 11 the following result can be obtained for topological posets whose topology is induced by a metric.

Corollary 12 Let $(X, \tau, \leq)$ be a topological poset and let $d$ be a metric on $X$ such that $\tau=\tau(d)$ and $(X, \tau(d), \leq)$ is $\leq-\tau(d)$-complete. Assume that the following assertions hold:

(1) There exists $x_{0} \in X$ such that $f\left(x_{0}\right) \leq x_{0}$.

(2) $f$ is monotone- $\leq$-continuous.

(3) $(X, \tau(d), \leq)$ has the orbital decreasing convergence property with respect to $f$.

Then $f$ has a unique fixed point in $\downarrow \leq x_{0}$.

Proof According to [12], every metric space is a balanced extended quasi-metric space. Thus the thesis is derived from Corollary 11.

Note that examples of topological posets whose topology is induced by a metric and, in addition, which are $\leq-\tau$-complete can be obtained from weightable quasi-metric spaces in the sense of [13] (see also [14]). Let us recall that a quasi-metric space $(X, d)$ is called weightable provided the existence of a mapping (weight) $w: X \rightarrow \mathbb{R}^{+}$such that

$$
d(x, y)+w(x)=d(y, x)+w(y)
$$

for all $x, y \in X$. Moreover, a quasi-metric space $(X, d)$ is bicomplete provided that the metric space $\left(X, d^{s}\right)$ is complete, where the metric $d^{s}$ is defined on $X$ by $d^{s}(x, y)=$ $\max \{d(x, y), d(y, x)\}$ for all $x, y \in X$. Furthermore, on account of [13], from a quasi-metric space $(X, d)$ a partial order $\leq_{d}$ can be defined on $X$ as follows: $x \leq_{d} y \Leftrightarrow d(x, y)=0$. From [13] it is not hard to check that, if $(X, d)$ is a bicomplete weightable quasi-metric space, then $\left(X, \tau\left(d^{s}\right), \leq_{d}^{-1}\right)$ is $\leq_{d}^{-1}-\tau\left(d^{s}\right)$-complete, where $\leq_{d}^{-1}$ denotes the dual partial order of $\leq_{d}$, i.e., $x \leq_{d}^{-1} y \Leftrightarrow y \leq_{d} x$ (see [1]).

The following are two instances of the aforesaid metric spaces.

Example 13 Consider the so-called upper quasi-metric space $\left(\mathbb{R}^{+}, d_{u}\right)$, where $d_{u}(x, y)=$ $\max \{y-x, 0\}$ for all $x, y \in \mathbb{R}^{+}$. According to [14], the upper quasi-metric space is weightable, with weight $w(x)=x$ for all $x \in \mathbb{R}^{+}$, and bicomplete. Moreover, $d_{u}^{s}(x, y)=|y-x|$ for all $x, y \in \mathbb{R}^{+}$. It is clear that $\left(\mathbb{R}^{+}, \tau(|\cdot|), \leq_{d_{u}}^{-1}\right)$ is $\leq_{d_{u}}^{-1}-\tau(|\cdot|)$-complete. In addition, consider the function $f$ from $\mathbb{R}^{+}$into itself given by $f(x)=\frac{x}{2}$. Then it is not hard to check that $\left(\mathbb{R}^{+}, \tau(|\cdot|), \leq_{d_{u}}^{-1}\right)$ has the orbital decreasing convergence property with respect to $f$.

Example 14 Let $\Sigma$ be a nonempty set (an alphabet). Denote by $\Sigma^{\infty}$ the set of all finite and infinite sequences (words) over $\Sigma$. For each $x \in \Sigma^{\infty}$ we denote by $l(x)$ the length of $x$. Hence $l(x) \in[1, \infty]$. We will write $x:=x_{1} x_{2} \cdots$, provided that $x \in \Sigma^{\infty}$ with $l(x)=\infty$, and we will write $x:=x_{1} x_{2} \cdots x_{n}$ whenever $x \in \Sigma^{\infty}$ with $l(x)=n<\infty$. Given $x, y \in \Sigma^{\infty}$, denote 
by $l(x, y)$ the length of the longest common prefix of $x$ and $y$, i.e. $l(x, y)=\sup \left\{n \in \mathbb{N}: x_{k}=\right.$ $y_{k}$ whenever $\left.k \leq n\right\}$ if $x$ and $y$ have a common prefix, and $l(x, y)=0$ otherwise. According to [13] and [14], $\Sigma^{\infty}$ can be equipped with the quasi-metric $d_{\Sigma^{\infty}}$ defined by

$$
d_{\Sigma^{\infty}}(x, y)=2^{-l(x, y)}-2^{-l(x)}
$$

for all $x, y \in \Sigma^{\infty}$. Of course the convention is adopted that $2^{-\infty}=0$. The quasi-metric space $\left(\Sigma^{\infty}, d_{\Sigma^{\infty}}\right)$ is weightable, with weight $w(x)=2^{-l(x)}$ for all $x \in \Sigma^{\infty}$, and bicomplete. Moreover, $x \leq_{d_{\Sigma} \infty}^{-1} y \Leftrightarrow y$ is a prefix of $x$. Then $\left(\Sigma^{\infty}, \tau\left(d_{\Sigma^{\infty}}^{s}\right), \leq_{d_{\Sigma} \infty}^{-1}\right)$ is $\leq_{d_{\Sigma} \infty}^{-1}-\tau\left(d_{\Sigma^{\infty}}^{s}\right)$-complete. Finally, consider $a \in \Sigma^{\infty}$ with $l(a)=1$ and define the function $f_{a}$ from $\Sigma^{\infty}$ into itself given by $f_{a}(x)=a x$, where by $a x$ we denote the concatenation of $a$ and $x$. Then it is not hard to check that $\left(\Sigma^{\infty}, \tau\left(d_{\Sigma^{\infty}}^{s}\right), \leq_{d_{\Sigma}^{\infty}}^{-1}\right)$ has the orbital decreasing convergence property with respect to $f_{a}$.

Notice that Corollary 12 is a fixed point result in the spirit of [11]. However, Corollary 12 provides something more than those results, concretely Proposition 2 and Theorem 1, given in [11]. Specifically, Corollary 12 yields the existence and uniqueness of the fixed point while Proposition 2 and Theorem 1 in [11] only guarantee the existence of the fixed point.

In order to introduce two new fixed point results we will need the involved extended quasi-metrics to enjoy a special property. Thus, if $(X, \tau, \leq)$ is a topological poset such that the topology $\tau$ is induced by an extended quasi-metric $d$ defined on $X$, then we will say that $(X, \tau, \leq)$ has the finite- $\leq$ property provided that $d(x, y) \neq \infty \Leftrightarrow x \leq y$. An example of this kind of topological posets is given by Example 2.

In the light of the preceding notion we provide a few fixed point results which incorporate contractive conditions for the self-mappings.

Corollary 15 Let $(X, \tau, \leq)$ be a topological poset and let $d$ be an extended quasi-metric on $X$ such that $\tau=\tau(d),(X, \tau(d), \leq)$ has the finite- $\leq$ property and is $\leq-\tau(d)$-complete. Assume that the following assertions hold:

(1) $(X, \tau(d))$ is Hausdorff.

(2) There exists $x_{0} \in X$ such that $d\left(f\left(x_{0}\right), x_{0}\right) \neq \infty$.

(3) For all $x, y \in X$ with $x \leq y$,

$$
d(f(x), f(y)) \leq d(x, y)
$$

(4) $(X, \tau(d), \leq)$ has the orbital decreasing convergence property with respect to $f$. Then $f$ has a unique fixed point in $\downarrow \leq x_{0}$.

Proof Since $(X, \tau, \leq)$ has the finite- $\leq$ property we see that $d\left(f\left(x_{0}\right), x_{0}\right) \neq \infty$ implies that $f\left(x_{0}\right) \leq x_{0}$. Next we prove that a mapping satisfying the contractive condition (1) is monotone- $\leq$-continuous. First of all we show the monotonicity of $f$. To this aim, let $x, y \in X$ with $x \leq y$. Then $d(x, y) \neq \infty$. Thus $d(f(x), f(y)) \neq \infty$. So $f(x) \leq f(y)$. Now suppose that there exist $z, x \in X$ with the sequence $\left(f^{n}(z)\right)_{n \in \mathbb{N}}$ decreasing and $x$ a lower bound of it such that $\left(f^{n}(z)\right)_{n \in \mathbb{N}}$ converges to $x$ with respect to $\tau$. Then $f(x) \leq f^{n}(z)$ and $d\left(f(x), f^{n+1}(z)\right) \leq d\left(x, f^{n}(z)\right)$ for all $n \in \mathbb{N}$. Hence $\left(f^{n+1}(z)\right)_{n \in \mathbb{N}}$ converges to $f(x)$ with respect to $\tau$. So $f$ is monotone- $\leq$-continuous. From Theorem 1 we deduce that $f$ has a fixed 
point in $\downarrow \leq x_{0}$. Assumption (4) and Theorem 9 give the uniqueness of the fixed point in $\downarrow \leq x_{0}$.

Example 10 shows that the orbital decreasing property cannot be omitted in the statement of Corollary 15 in order to guarantee the uniqueness of the fixed point.

Corollary 16 Let $(X, \tau, \leq)$ be a topological poset and let $d$ be an extended quasi-metric on $X$ such that $\tau=\tau(d),(X, \tau(d), \leq)$ has the finite- $\leq$ property and is $\leq-\tau(d)$-complete. Assume that the following assertions hold:

(1) $(X, \tau(d))$ is Hausdorff.

(2) There exists $x_{0} \in X$ such that $d\left(f\left(x_{0}\right), x_{0}\right) \neq \infty$.

(3) There exists $c \in[0,1[$ such that

$$
d(f(x), f(y)) \leq c d(x, y)
$$

for all $x, y \in X$ with $x \leq y$.

(4) $(X, \tau(d), \leq)$ has the orbital decreasing convergence property with respect to $f$.

Then $f$ has a unique fixed point in $\downarrow \leq x_{0}$.

It should be stressed that Corollary 16 is related to Theorem 5 in [6].

In the next fixed point result we show that the monotonicity assumed in Theorem 1 can be replaced by a special kind of contractive condition.

Theorem 17 Let $(X, \tau, \leq)$ be a topological poset and let $d$ be an extended quasi-metric on $X$ such that $\tau=\tau(d),(X, \tau(d), \leq)$ is $\leq-\tau(d)$-complete and holds the finite- $\leq$ property. Assume that the following assertions hold:

(1) $(X, \tau(d))$ is Hausdorff.

(2) There exists $x_{0} \in X$ such that $d\left(f\left(x_{0}\right), x_{0}\right) \neq \infty$.

(3) For all $n \in \mathbb{N}$,

$$
d\left(f^{n+1}\left(x_{0}\right), f^{n}\left(x_{0}\right)\right) \leq d\left(f^{n}\left(x_{0}\right), f^{n-1}\left(x_{0}\right)\right)
$$

(4) If $z, x \in X$ and the sequence $\left(f^{n}(z)\right)_{n \in \mathbb{N}}$ is decreasing with $x$ as a lower bound such that $\left(f^{n}(z)\right)_{n \in \mathbb{N}}$ converges to $x$ with respect to $\tau(d)$, then the sequence $\left(f^{n+1}(z)\right)_{n \in \mathbb{N}}$ converges to $f(x)$ with respect to $\tau(d)$.

(5) $(X, \tau(d), \leq)$ has the orbital decreasing convergence property with respect to $f$.

Then $f$ has a unique fixed point in $\downarrow \leq x_{0}$.

Proof From (3) we obtain that $d\left(f^{n+1}\left(x_{0}\right), f^{n}\left(x_{0}\right)\right) \neq \infty$ for all $n \in \mathbb{N}$, since $d\left(f\left(x_{0}\right), x_{0}\right) \neq \infty$. The fact that $(X, \tau(d), \leq)$ has the finite- $\leq$ property shows that the sequence $\left(f^{n}\left(x_{0}\right)\right)_{n \in \mathbb{N}}$ is decreasing. Since $(X, \tau(d), \leq)$ is $\leq-\tau(d)$-complete we deduce the existence of a lower bound $x^{*}$ of $\left(f^{n}\left(x_{0}\right)\right)_{n \in \mathbb{N}}$ such that $\left(f^{n}\left(x_{0}\right)\right)_{n \in \mathbb{N}}$ converges to $x^{*}$ with respect to $\tau(d)$. Whence we have $x^{*} \in \downarrow \leq x_{0}$. By assumption (4) we see that $\left(f^{n+1}\left(x_{0}\right)\right)_{n \in \mathbb{N}}$ converges to $f\left(x^{*}\right)$ with respect to $\tau(d)$. The fact that $(X, \tau(d))$ is Hausdorff provides us with the result that $x^{*}$ is a fixed point of $f$. Finally, assumption (5) shows the uniqueness of the fixed point in $\downarrow \leq x_{0}$ following the same argument as those given in Theorem 9. 
Once again, Example 10 shows that Theorem 17 does not guarantee the uniqueness of the fixed point if the orbital decreasing property is omitted in the statement of Theorem 17. Note that Corollary 15 can be retrieved as a particular case from Theorem 17.

Corollary 18 Let $(X, \tau, \leq)$ be a topological poset and let $d$ be an extended quasi-metric on $X$ such that $\tau=\tau(d),(X, \tau(d), \leq)$ is $\leq-\tau(d)$-complete and holds the finite- $\leq$ property. Assume that the following assertions hold:

(1) $(X, \tau(d))$ is Hausdorff.

(2) There exists $x_{0} \in X$ such that $d\left(f\left(x_{0}\right), x_{0}\right) \neq \infty$.

(3) For all $n \in \mathbb{N}$,

$$
d\left(f^{n+1}\left(x_{0}\right), f^{n}\left(x_{0}\right)\right) \leq c d\left(f^{n}\left(x_{0}\right), f^{n-1}\left(x_{0}\right)\right) .
$$

(4) $f$ is continuous from $(X, \tau(d))$ into itself.

(5) $(X, \tau(d), \leq)$ has the orbital decreasing convergence property with respect to $f$.

Then $f$ has a unique fixed point in $\downarrow \leq x_{0}$.

Proof Obviously every continuous mapping satisfies assertion (4) in the statement of Corollary 17. So the thesis of the result follows from the aforesaid corollary.

It should be stressed that Corollary 18 is related to Theorem 3 in [15].

We end the section proving that the topological posets under consideration in the statement of Corollary 15 and Theorem 17 belong to the special class of right $K$-sequentially complete extended quasi-metric spaces. Let us recall that, following [6], an extended quasi-metric space $(X, d)$ is right $K$-sequentially complete whenever every right $K$-Cauchy sequence is convergent with respect to $\tau(d)$, where a sequence $\left(x_{n}\right)_{n \in \mathbb{N}}$ is right $K$-Cauchy if for each $\varepsilon>0$ there is $n_{0} \in \mathbb{N}$ such that $d\left(x_{m}, x_{n}\right)<\varepsilon$ for all $m \geq n \geq n_{0}$.

Proposition 19 Let $(X, \tau, \leq)$ be a topological poset and let $d$ be an extended quasi-metric on $X$ such that $\tau=\tau(d)$ and $(X, \tau(d), \leq)$ holds the finite- $\leq$ property. If $(X, \tau(d), \leq)$ is $\leq-$ $\tau(d)$-complete, then $(X, d)$ is right $K$-sequentially complete.

Proof Let $\left(x_{n}\right)_{n \in \mathbb{N}}$ be a right $K$-Cauchy sequence. Then there exists $n_{0} \in \mathbb{N}$ such that $d\left(x_{m}, x_{n}\right)<1$ for all $n \geq n_{0}$. Since $(X, d)$ has the finite- $\leq$ property and $d\left(x_{m}, x_{n}\right) \neq \infty$ for all $m \geq n \geq n_{0}$ we have $x_{n+1} \leq x_{n}$ for all $n \geq n_{0}$. It follows that $\left(x_{n}\right)_{n \geq n_{0}}$ is a decreasing sequence in $(X, \leq)$. The fact that $(X, \tau, \leq)$ is $\leq-\tau(d)$-complete guarantees the existence of a lower bound $x$ of $\left(x_{n}\right)_{n \geq n_{0}}$ such that $\left(x_{n}\right)_{n \geq n_{0}}$ converge to $x$ with respect to $\tau(d)$. Consequently $(X, d)$ is right $K$-sequentially complete.

The converse of Proposition 19 does not hold, as the next example shows.

Example 20 It is not hard to see that the topological poset $\left(\mathbb{R}_{0}^{+}, \tau\left(d_{S}\right), \preceq\right)$ introduced in Example 2 has the finite- $\preceq$ property. Moreover, $\left(\mathbb{R}_{0}^{+}, d_{S}\right)$ is right $K$-sequentially complete. However, it has been shown in the aforesaid example that $\left(\mathbb{R}_{0}^{+}, \tau\left(d_{S}\right), \preceq\right)$ is not $\preceq-\tau\left(d_{S}\right)$ complete.

The following example shows that the finite- $\leq$ property of the extended quasi-metric space cannot be deleted in the statement of Proposition 19. 
Example 21 Consider the topological poset $\left(\mathbb{R}_{1}^{+}, \tau\left(d_{\infty}\right), \leq\right)$ introduced in Example 3. Of course $\left(\mathbb{R}_{1}^{+}, \tau\left(d_{\infty}\right), \leq\right)$ is $\leq-\tau\left(d_{\infty}\right)$-complete. However, $\left(\mathbb{R}_{1}^{+}, d_{\infty}\right)$ is not right $K$-sequentially complete. Indeed, the sequence $\left(x_{n}\right)_{n \in \mathbb{N}}$ such that $x_{n}=n$ for all $n \in \mathbb{N}$ is right $K$-Cauchy but it does not converge to any $x \in \mathbb{R}_{1}^{+}$. Clearly $\left(\mathbb{R}_{1}^{+}, \tau\left(d_{\infty}\right), \leq\right)$ has not the finite- $\leq$ property.

\section{The application}

In computer science, the running time of computing of an algorithm $A$ is denoted by a function $T^{A}$ defined from $\mathbb{N}$ into $(0, \infty)$. So the time taken by the algorithm to solve the problem for which it has been designed is denoted by $T^{A}(n)$, where $n \in \mathbb{N}$ represents the size of the input data to be processed.

When the running time of computing of an algorithm has to be determined, the engineers do not provide the exact expression of function that represents such a time. In contrast, they work with the so-called asymptotic complexity classes. In general, they provide an upper bound for $T_{A}(n)$ whenever the input size $n$ is large enough (observe that, from a computing point of view, the interesting cases are the asymptotic ones, i.e., those in which the algorithm processes input data of large size). From now on, we denote by $\mathcal{T C}$ the set of functions $\{f: \mathbb{N} \rightarrow(0, \infty)\}$. Although we assume that the reader is familiar with the basic notions from asymptotic complexity analysis (for a full treatment we refer the reader to [16]), let us recall that $g$ is an asymptotic upper bound of $f$, denoted by $f \in \mathcal{O}(g)$, provided that there exist $n_{0} \in \mathbb{N}$ and $c \in \mathbb{R}_{0}^{+}$such that $f(n) \leq c g(n)$ for all $n \in \mathbb{N}$ with $n \geq n_{0}$.

In many situations the running time of computing of the algorithm under consideration satisfies a recurrence equation as the following one:

$$
T(n)= \begin{cases}c & \text { if } n=1, \\ a(n) T(n-1)+h(n) & \text { if } n \geq 2,\end{cases}
$$

where $c \in \mathbb{R}_{0}^{+}, a, h \in \mathcal{T C}$, and $\sup _{n \in \mathbb{N}} a(n)<\infty$.

Note that the preceding recurrence equation admits only one solution. Hence such a solution represents the running time of computing of the algorithm under consideration in each case. So, from engineering viewpoint, it remains to provide the asymptotic upper bound of the solution to the preceding recurrence equation. Note that the solution itself is not important. The only really important fact about the solution to the recurrence equation is the asymptotic bound because it delimits the maximum time spent by the algorithm in order to yield a solution to the problem.

In 2003, a formal method was given in [6] to discuss the running time of computing of algorithms that belong to the divide and conquer class. The development of the aforementioned method is based on the use of an extended quasi-metric space, the so-called extended dual complexity space, introduced in the aforesaid reference. Concretely, the extended dual complexity space is formed by the pair $\left(\mathcal{C}, e_{\mathcal{C}}\right)$, where

$$
\mathcal{C}=\left\{f \in \mathcal{T C}: \sum_{n=1}^{\infty} 2^{-n} f(n)<\infty\right\}
$$

and $e_{\mathcal{C}}$ is the extended quasi-metric given by

$$
e_{\mathcal{C}}(f, g)= \begin{cases}\sum_{n=1}^{\infty} 2^{-n}(g(n)-f(n)) & \text { if } f \preceq_{\mathcal{C}} g \\ \infty & \text { if otherwise }\end{cases}
$$


Of course, the partial order $\preceq_{\mathcal{C}}$ is defined on $\mathcal{C}$ by $f \preceq_{\mathcal{C}} g \Leftrightarrow f(n) \leq g(n)$ for all $n \in \mathbb{N}$. It must be pointed out that the convergence condition ' $\sum_{n=1}^{\infty} 2^{-n} f(n)<\infty$ ' in the definition of $\mathcal{C}$ is not restrictive from a complexity point of view.

In [6], it was proved that the extended dual complexity space enjoys the following interesting properties, which played a relevant role in the aforementioned method.

(1) $\left(\mathcal{C}, \tau\left(e_{\mathcal{C}}\right)\right)$ is Hausdorff. In fact, $\left(\mathcal{C}, e_{\mathcal{C}}\right)$ is balanced (Theorem 2 in [6]).

(2) Every decreasing sequence $\left(f_{n}\right)_{n \in \mathbb{N}}$ in $(\mathcal{C}, \preceq)$ has an infimum $f$ to which it converges with respect to $\tau\left(e_{\mathcal{C}}\right)$ (Theorem 3 in [6]).

At this point, we observe that the above relevant properties establish that $\left(\mathcal{C}, e_{\mathcal{C}}, \preceq_{\mathcal{C}}\right)$ is a meet $\preceq_{\mathcal{C}}-\tau\left(e_{\mathcal{C}}\right)$-complete topological poset. Moreover, $e_{\mathcal{C}}(f, g) \neq \infty \Leftrightarrow f \preceq_{\mathcal{C}} g$ and, thus, $\left(\mathcal{C}, e_{\mathcal{C}}, \preceq_{\mathcal{C}}\right)$ has the finite- $\preceq_{\mathcal{C}}$ property. Furthermore, we have the following.

Inspired, on the one hand, by the fact that the above relevant properties of the extended dual complexity space, properties (1) and (2), can be reclaimed in our general framework of topological posets and, on the other hand, by the fact that the running time of those algorithms treated in [6] holds recurrence equations that can be retrieved as a particular case of the recurrence equation (5), in the following we are interested in providing a formal method based on our abstract approach of topological posets, i.e. on Theorems 1 , in such a way that we are able to determine the asymptotic upper bound of those algorithms whose running time of computing satisfies (5).

Consider the mapping $\Phi_{T}$ from $\mathcal{C}$ into itself defined as follows:

$$
\Phi_{T}(f)(n)= \begin{cases}c & \text { if } n=1 \\ a(n) f(n-1)+h(n) & \text { if } n \geq 2 .\end{cases}
$$

It is clear that the fixed points of $\Phi_{T}$ are the solutions to the recurrence equation (5). Moreover, it is easy to check that if $\Phi_{T}$ has a fixed point, then it is unique (it can be proved by induction).

The next result introduces our new methodology.

Proposition 22 Let $\Phi_{T}$ be the mapping from $\mathcal{C}$ into itself given by (7). If there exists $g \in \mathcal{C}$ such that $\Phi(g) \preceq_{\mathcal{C}} g$, then $f \in \mathcal{O}(g)$.

Proof A straightforward computation shows that $\Phi_{T}$ is a monotone- $\preceq_{\mathcal{C}}$-continuous mapping. Moreover, we have seen that $\left(\mathcal{C}, \tau\left(e_{\mathcal{C}}\right)\right)$ is Hausdorff and that $\left(\mathcal{C}, e_{\mathcal{C}}, \preceq_{\mathcal{C}}\right)$ is a meet $\preceq_{\mathcal{C}}-\tau\left(e_{\mathcal{C}}\right)$-complete topological poset. So if there exists $g \in \mathcal{C}$ such that $\Phi(g) \preceq_{\mathcal{C}} g$, then Theorem 1 gives the existence of a fixed point $f^{*}$ of $\Phi$ such that $f^{*} \preceq_{\mathcal{C}} g$. It follows that $f^{*} \in \mathcal{O}(g)$.

Observe that the mapping $\Phi$ has only a fixed point. Hence the fixed point guaranteed by Proposition 22, $f^{*}$ represents the solution to the recurrence equation (5) and, thus, the running time of computing of the algorithm under consideration. So the aforesaid proposition gives a methodology to provide the asymptotic upper bound of running time of computing of those algorithms whose time of computing is the solution to (5).

In order to illustrate the method provided by Proposition 22 let us consider the quicksort algorithm. When we consider the average cost analysis, the running time of computing of 
the aforementioned algorithm is the solution to the recurrence equation below [17]:

$$
Q(n)= \begin{cases}c & \text { if } n=1 \\ \frac{n+1}{n} Q(n-1)+2 & \text { if } n \geq 2\end{cases}
$$

where $c \in \mathbb{R}_{0}^{+}$. Of course, the preceding recurrence equation can be recovered from (5) when we take $h(n)=2$ and $a(n)=\frac{n+1}{n}$ for all $n \in \mathbb{N}$. Hence the solution to (8) is the (unique) fixed point of $\Phi_{Q}$, where $\Phi_{Q}$ is defined by (7) with $h(n)=2$ and $a(n)=\frac{n+1}{n}$ for all $n \in \mathbb{N}$. A simple computation yields $\Phi_{Q}\left(g_{0}\right) \preceq_{\mathcal{C}} g_{0}$ for any $g_{0} \in \mathcal{C} \Leftrightarrow g_{0}$ that satisfies the following:

$$
g_{0}(n) \geq \begin{cases}2+\frac{3}{2} c & \text { if } n=1, \\ \frac{c}{2}(n+1)+2(n+1) \sum_{j=1}^{n-1} \frac{1}{j+2} & \text { if } n \geq 2 .\end{cases}
$$

Hence taking $f_{0} \in \mathcal{C}$ defined by

$$
f_{0}(n)= \begin{cases}2+\frac{3}{2} c & \text { if } n=1, \\ \frac{c}{2}(n+1)+2(n+1) \sum_{j=1}^{n-1} \frac{1}{j+2} & \text { if } n \geq 2,\end{cases}
$$

we have $\Phi_{Q}\left(f_{0}\right) \preceq_{\mathcal{C}} f_{0}$ and, thus $f^{*} \in \mathcal{O}\left(f_{0}\right)$. Besides, according to [17], $f_{0} \in \mathcal{O}(g)$, where

$$
g(n)= \begin{cases}2+\frac{3}{2} c & \text { if } n=1 \\ n \log _{2}(n) & \text { if } n \geq 2\end{cases}
$$

It follows that $f^{*} \in \mathcal{O}(g)$, where $g$ is defined by (11).

After confirming the validity of the new fixed point technique for obtaining asymptotic upper bounds in complexity analysis of algorithms, we end the paper noting that the new method based on Theorem 1 presents an advantage with respect to that used in [6]. Specifically, the new methodology does not require one to impose contractive conditions (compare Theorem 5 in [6]) and, in addition, it provides information as regards the asymptotic upper bound of the running time of computing. Finally, notice that the fixed point technique developed in [6] uses the fact that the extended dual complexity space $\left(\mathcal{C}, e_{\mathcal{C}}\right)$ is right $K$-sequentially complete (Theorem 4 in [6]). Again such a property also can be recovered from our approach. In fact, the completion follows from the fact that $\left(\mathcal{C}, e_{\mathcal{C}}, \preceq_{\mathcal{C}}\right)$ is a $\preceq_{\mathcal{C}}$ $\tau\left(e_{\mathcal{C}}\right)$-complete topological poset with the finite- $\preceq_{\mathcal{C}}$ property and Proposition 19.

Competing interests

The authors declare that they have no competing interests.

Authors' contributions

All authors contributed equally and significantly in writing this article. All authors read and approved the final manuscript.

\section{Author details}

1 Operator Theory and Applications Research Group, Department of Mathematics, King Abdulaziz University, P.O. Box 80203, Jeddah, 21859, Saudi Arabia. ${ }^{2}$ Department of Mathematics and Computer Science, University of Balearic Islands, Ctra. de Valldemossa km. 7.5, Palma de Mallorca, 07122, Spain. ${ }^{3}$ Department of Mathematics, Faculty of Science AL Faisaliah Campus, King Abdulaziz University, P.O. Box 4087, Jeddah, 21491, Saudi Arabia.

\section{Acknowledgements}

This project was funded by the National Plan for Science, Technology and Innovation (MAARIFAH), King Abdulaziz City for Science and Technology, the Kingdom of Saudi Arabia; award number (12-MAT051-03). The authors also acknowledge with thanks the Science and Technology Unit, King Abdulaziz University, for technical support. The authors are grateful to three anonymous referees for their useful suggestions and comments. 


\section{References}

1. Davey, BA, Priestley, HA: Introduction to Lattices and Order. Cambridge University Press, Cambridge (1990)

2. Scott, DS: Outline of a mathematical theory of computation. In: Proc. 4th Annual Princeton Conference on Information Sciences and Systems, pp. 169-176 (1970)

3. Hitzler, P, Seda, AK: Generalized distance functions in the theory of computation. Comput. J. 53, 443-464 (2010)

4. Hitzler, P, Seda, AK: Mathematical Aspects of Logic Programming Semantics. CRC Press, Boca Raton (2011)

5. Schellekens, M: The Smyth completion: a common foundation for the denotational semantics and complexity analysis. Electron. Notes Theor. Comput. Sci. 1, 211-232 (1995)

6. Romaguera, S, Sánchez-Pérez, EA, Valero, O: Computing complexity distances between algorithms. Kybernetika 39 569-582 (2003)

7. Alghamdi, MA, Shahzad, N, Valero, O: New results on the Baire partial quasi-metric space, fixed point theory and asymptotic complexity analysis for recursive programs. Fixed Point Theory Appl. 2014, Article ID 14 (2014)

8. Alghamdi, MA, Shahzad, N, Valero, O: Fixed point theorems in generalized metric spaces with applications to computer science. Fixed Point Theory Appl. 2013, Article ID 118 (2013)

9. Shahzad, N, Valero, O: On 0-complete partial metric spaces and quantitative fixed point techniques in denotational semantics. Abstr. Appl. Anal. 2013, Article ID 985095 (2013)

10. Künzi, HPA: Nonsymmetric topology. In: Proc. Colloquium on Topology, Szekszárd, Hungary, 1993. Colloq. Math. Soc János Bolyai Math. Studies, vol. 4, pp. 303-338 (1995)

11. Granas, A, Horvath, CD: On the order-theoretic Cantor theorem. Taiwan. J. Math. 4, 203-213 (2000)

12. Doitchinov, D: On completeness in quasi-metric spaces. Topol. Appl. 30, 127-148 (1988)

13. Matthews, SG: Partial metric topology. Ann. N.Y. Acad. Sci. 728, 183-197 (1994)

14. Oltra, S, Romaguera, S, Sánchez-Pérez, EA: Bicompleting weightable quasi-metric spaces and partial metric spaces. Rend. Circ. Mat. Palermo 51, 151-162 (2002)

15. Rodríguez-López, J, Romaguera, S, Valero, O: Denotational semantics for programming languages, balanced quasi-metrics and fixed points. Int. J. Comput. Math. 85, 623-630 (2008)

16. Brassard, G, Bratley, P: Algorithms: Theory and Practice. Prentice Hall, Englewood Cliffs (1988)

17. Cull, P, Flahive, M, Robson, R: Difference Equations: From Rabbits to Chaos. Springer, New York (2005)

\section{Submit your manuscript to a SpringerOpen ${ }^{\circ}$ journal and benefit from:}

- Convenient online submission

- Rigorous peer review

- Immediate publication on acceptance

- Open access: articles freely available online

- High visibility within the field

- Retaining the copyright to your article 